\title{
A New Class of Banach Spaces and Its Relation with Some Geometric Properties of Banach Spaces
}

\author{
M. Salimi and S. M. Moshtaghioun \\ Department of Mathematics, Yazd University, Yazd 89195-741, Iran \\ Correspondence should be addressed to S. M. Moshtaghioun, moshtagh@yazduni.ac.ir
}

Received 29 December 2011; Accepted 14 February 2012

Academic Editor: Saminathan Ponnusamy

Copyright (C) 2012 M. Salimi and S. M. Moshtaghioun. This is an open access article distributed under the Creative Commons Attribution License, which permits unrestricted use, distribution, and reproduction in any medium, provided the original work is properly cited.

By introducing the concept of $L$-limited sets and then $L$-limited Banach spaces, we obtain some characterizations of it with respect to some well-known geometric properties of Banach spaces, such as Grothendieck property, Gelfand-Phillips property, and reciprocal Dunford-Pettis property. Some complementability of operators on such Banach spaces are also investigated.

\section{Introduction and Preliminaries}

A subset $A$ of a Banach space $X$ is called limited (resp., Dunford-Pettis (DP)), if every weak* null (resp., weak null) sequence $\left(x_{n}^{*}\right)$ in $X^{*}$ converges uniformly on $A$, that is,

$$
\lim _{n \rightarrow \infty} \sup _{a \in A}\left|\left\langle a, x_{n}^{*}\right\rangle\right|=0
$$

Also if $A \subseteq X^{*}$ and every weak null sequence $\left(x_{n}\right)$ in $X$ converges uniformly on $A$, we say that $A$ is an $L$-set.

We know that every relatively compact subset of $X$ is limited and clearly every limited set is DP and every DP subset of a dual Banach space is an $L$-set, but the converse of these assertions, in general, are false. If every limited subset of a Banach space $X$ is relatively compact, then $X$ has the Gelfand-Phillips property (GP). For example, the classical Banach spaces $c_{0}$ and $\ell_{1}$ have the GP property and every reflexive space, every Schur space (i.e., weak and norm convergence of sequences in $X$ coincide), and dual of spaces containing no copy of $\ell_{1}$ have the same property.

Recall that a Banach space $X$ is said to have the DP property if every weakly compact operator $T: X \rightarrow Y$ is completely continuous (i.e., $T$ maps weakly null sequences into norm 
null sequences) and $X$ is said to have the reciprocal Dunford-Pettis property (RDP) if every completely continuous operator on $X$ is weakly compact.

So the Banach space $X$ has the DP property if and only if every relatively weakly compact subset of $X$ is DP and it has the RDP property if and only if every $L$-set in $X^{*}$ is relatively weakly compact.

A stronger version of DP property was introduced by Borwein et al. in [1]. In fact, a Banach space $X$ has the DP* property if every relatively weakly compact subset of $X$ is limited. But if $X$ is a Grothendieck space (i.e., weak and weak* convergence of sequences in $X^{*}$ coincide), then these properties are the same on $X$. The reader can find some useful and additional properties of limited and DP sets and Banach spaces with the GP, DP, or RDP property in [2-6].

We recall from [7] that a bounded linear operator $T: X \rightarrow Y$ is limited completely continuous (lcc) if it carries limited and weakly null sequences in $X$ to norm null ones in $Y$. We denote the class of all limited completely continuous operators from $X$ to $Y$ by $\operatorname{Lcc}(X, Y)$. It is clear that every completely continuous operator is lcc and we showed in [7] that every weakly compact operator is limited completely continuous.

Here, by introducing a new class of subsets of Banach spaces that are called L-limited sets, we obtain some characterizations of Banach spaces that every L-limited set is relatively weakly compact and then we investigate the relation between these spaces with the GP, DP, RDP and Grothendieck properties.

The notations and terminologies are standard. We use the symbols $X, Y$, and $Z$ for arbitrary Banach spaces. We denoted the closed unit ball of $X$ by $B_{X}$, absolutely closed convex hull of a subset $A$ of $X$ by $a \overline{c o}(A)$, the dual of $X$ by $X^{*}$, and $T^{*}$ refers to the adjoint of the operator $T$. Also we use $\left\langle x, x^{*}\right\rangle$ for the duality between $x \in X$ and $x^{*} \in X^{*}$. We denote the class of all bounded linear, weakly compact, and completely continuous operators from $X$ to $Y$ by $L(X, Y), W(X, Y)$, and $C_{c}(X, Y)$ respectively. We refer the reader for undefined terminologies to the classical references $[8,9]$.

\section{L-Limited Sets}

Definition 2.1. A subset $A$ of dual space $X^{*}$ is called an $L$-limited set, if every weak null and limited sequence $\left(x_{n}\right)$ in $X$ converges uniformly on $A$.

It is clear that every $L$-set in $X^{*}$ is $L$-limited and every subset of an $L$-limited set is the same. Also, it is evident that every L-limited set is weak* bounded and so is bounded. The following theorem gives additional properties of these sets.

Theorem 2.2. (a) Absolutely closed convex hull of an L-limited set is L-limited.

(b) Relatively weakly compact subsets of dual Banach spaces are L-limited.

(c) Every weak* null sequence in dual Banach space is an L-limited set.

Proof. Let $A \subseteq X^{*}$ be an L-limited set, and the sequence $\left(x_{n}\right)$ in $X$ is weak null and limited. Since

$$
\sup \left\{\left|\left\langle x_{n}, x^{*}\right\rangle\right|: x^{*} \in a \overline{\mathrm{co}}(A)\right\}=\sup \left\{\left|\left\langle x_{n}, x^{*}\right\rangle\right|: x^{*} \in A\right\},
$$


the proof of (a) is clear. For the proof of (b) suppose $A \subset X^{*}$ is relatively weakly compact but it is not an $L$-limited set. Then there exists a weakly null and limited sequence $\left(x_{n}\right)$ in $X$, a sequence $\left(a_{n}\right)$ in $A$ and an $\epsilon>0$ such that $\left|\left\langle x_{n}, a_{n}\right\rangle\right|>\epsilon$ for all integer $n$. Since $A$ is relatively weakly compact, there exists a subsequence $\left(a_{n_{k}}\right)$ of $\left(a_{n}\right)$ that converges weakly to an element $a \in X^{*}$. Since

$$
\left|\left\langle x_{n_{k}}, a_{n_{k}}\right\rangle\right| \leq\left|\left\langle x_{n_{k}}, a_{n_{k}}-a\right\rangle\right|+\left|\left\langle x_{n_{k}}, a\right\rangle\right| \longrightarrow 0,
$$

we have a contradiction.

Finally, for (c), suppose $\left(x_{n}^{*}\right)$ is a weak* null sequence in $X^{*}$. Define the operator $T$ : $X \rightarrow c_{0}$ by $T(x)=\left(\left\langle x, x_{n}^{*}\right\rangle\right)$. Since $c_{0}$ has the GP property by [7], $T$ is lcc. So for each weakly null and limited sequence $\left(x_{m}\right)$ in $X$, we have

$$
\sup _{n}\left|\left\langle x_{m}, x_{n}^{*}\right\rangle\right|=\left\|T\left(x_{m}\right)\right\| \longrightarrow 0,
$$

as $m \rightarrow \infty$. Hence $\left(x_{n}^{*}\right)$ is an L-limited set.

Note that the converse of assertion (b) in general is false. In fact, the following theorem show that the closed unit ball of $\ell_{1}$ is an L-limited set, while the standard unit vectors $\left(e_{n}\right)$ in $c_{0}$, as a weakly null sequence, shows that the closed unit ball of $\ell_{1}$ is neither an $L$-set nor a relatively weakly compact. The following Theorem 2.4 , give a necessary and sufficient condition for Banach spaces that $L$-sets and $L$-limited sets in its dual coincide.

Theorem 2.3. A Banach space $X$ has the GP property if and only if every bounded subset of $X^{*}$ is an L-limited set.

Proof. Since the Banach space $X$ has the GP property if and only if every limited and weakly null sequence $\left(x_{n}\right)$ in $X$ is norm null [10], the proof is clear.

Theorem 2.4. A Banach space $X$ has the $D P^{*}$ property if and only if each L-limited set in $X^{*}$ is an L-set.

Proof. Suppose $X$ has the $\mathrm{DP}^{*}$ property. Since every weakly null sequence in $X$ is limited so every $L$-limited set in $X^{*}$ is $L$-set.

Conversely, it is enough to show that, for each Banach space $Y, C c(X, Y)=\operatorname{Lcc}(X, Y)$ [7, Theorem 2.8]. If $T: X \rightarrow Y$ is lcc, it is clear that $T^{*}\left(B_{Y^{*}}\right)$ is an $L$-limited set. So by hypothesis, it is an $L$-set and we know that the operator $T: X \rightarrow Y$ is completely continuous if and only if $T^{*}\left(B_{Y^{*}}\right)$ is an $L$-set.

The following two corollaries extend Theorem 3.3 and Corollary 3.4 of [1].

Corollary 2.5. For a Banach space $X$, the following are equivalent.

(a) X has the DP* property,

(b) If $Y$ has the Gelfand-Phillips property, then each operator $T: X \rightarrow Y$ is completely continuous. 
Proof. (a) $\Rightarrow$ (b). Suppose that $Y$ has the Gelfand-Phillips property. By [7, Theorem 2.2], every operator $T: X \rightarrow Y$ is lcc, thus $T^{*}\left(B_{Y^{*}}\right)$ is an $L$-limited set and by Theorem 2.3, it is an $L$-set. Hence $T$ is completely continuous.

(b) $\Rightarrow$ (a). If $X$ does not have the DP* property, there exists a weakly null sequence $\left(x_{n}\right)$ in $X$ that is not limited. So there is a weak * null sequence $\left(x_{n}^{*}\right)$ in $X^{*}$ such that $\left|\left\langle x_{n}, x_{n}^{*}\right\rangle\right|>\epsilon$, for all integer $n$ and some positive $\epsilon$ [10]. Now the bounded operator $T: X \rightarrow c_{0}$ defined by $T(x)=\left(\left\langle x, x_{n}^{*}\right\rangle\right)$ is not completely continuous, since $\left(x_{n}\right)$ is weakly null and $\left\|T x_{n}\right\|>\epsilon$ for all $n$. This is a contradiction.

Corollary 2.6. A Gelfand-Phillips space has the DP* property if and only if it has the Schur property.

Proof. It is clear that the Banach space $X$ has the Schur property if and only if every bounded subset of $X^{*}$ is an L-set. Now, if $X$ is a GP space with the DP* property, then by Theorem 2.3, unit ball $X^{*}$ is $L$-limited and so it is an $L$-set. The converse of the assertion is also clear.

Definition 2.7. A Banach space $X$ has the L-limited property, if every L-limited set in $X^{*}$ is relatively weakly compact.

Theorem 2.8. For a Banach space $X$, the following are equivalent:

(a) X has the L-limited property,

(b) for each Banach space $Y, \operatorname{Lcc}(X, Y)=W(X, Y)$,

(c) $\operatorname{Lcc}\left(X, \ell_{\infty}\right)=W\left(X, \ell_{\infty}\right)$.

Proof. (a) $\Rightarrow$ (b). Suppose that $X$ has the L-limited property and $T: X \rightarrow Y$ is lcc. Thus $T^{*}\left(B_{Y^{*}}\right)$ is an $L$-limited set in $X^{*}$. So by hypothesis, it is relatively weakly compact and $T$ is a weakly compact operator.

(b) $\Rightarrow$ (c). It is clear.

(c) $\Rightarrow$ (a). If $X$ does not have the $L$-limited property, there exists an $L$-limited subset $A$ of $X^{*}$ that is not relatively weakly compact. So there is a sequence $\left(x_{n}^{*}\right) \subseteq A$ with no weakly convergent subsequence. Now we show that the operator $T: X \rightarrow \ell_{\infty}$ defined by $T(x)=$ $\left(\left\langle x, x_{n}^{*}\right\rangle\right)$ for all $x \in X$ is limited completely continuous but it is not weakly compact. As $\left(x_{n}^{*}\right) \subseteq A$ is $L$-limited set, for every weakly null and limited sequence $\left(x_{m}\right)$ in $X$ we have

$$
\left\|T\left(x_{m}\right)\right\|=\sup _{n}\left|\left\langle x_{m}, x_{n}^{*}\right\rangle\right| \longrightarrow 0 \quad \text { as } m \longrightarrow \infty,
$$

thus $T$ is a limited completely continuous operator. It is easy to see that $T^{*}\left(e_{n}^{*}\right)=x_{n}^{*}$, for all $n \in \mathbb{N}$. Thus $T^{*}$ is not a weakly compact operator and neither is $T$. This finishes the proof.

The following corollary shows that the Banach spaces $c_{0}$ and $\ell_{1}$ do not have the Llimited property.

Corollary 2.9. A Gelfand-Phillips space has the L-limited property if and only if it is reflexive. 
Proof. If a Banach space $X$ has the GP property, then by [7], the identity operator on $X$ is lcc and so is weakly compact, thanks to the $L$-limited property of $X$. Hence $X$ is reflexive.

Theorem 2.10. If a Banach space X has the L-limited property, then it has the RDP and Grothendieck properties.

Proof. At the first, we show that $X$ has the RDP property. For arbitrary Banach space $Y$, let $T: X \rightarrow Y$ be a completely continuous operator. Thus it is limited completely continuous and so by Theorem 2.8, $T$ is weakly compact. Hence $X$ has the RDP property.

By [11], we know that a Banach space $X$ is Grothendieck if and only if $W\left(X, c_{0}\right)=$ $L\left(X, c_{0}\right)$. Since $c_{0}$ has the GP property, by [7], $\operatorname{Lcc}\left(X, c_{0}\right)=L\left(X, c_{0}\right)$ and by hypothesis on $X$, $W\left(X, c_{0}\right)=\operatorname{Lcc}\left(X, c_{0}\right)$. So $X$ is Grothendieck.

We do not know the converse of Theorem 2.10, in general, is true or false. In the following, we show that in Banach lattices that are Grothendieck and have the DP property, the converse of this theorem is correct.

Theorem 2.11. If a Banach lattice X has both properties of Grothendieck and DP, then it has the L-limited property.

Proof. Suppose that $T: X \rightarrow Y$ is limited completely continuous. We know, that in Grothendieck Banach spaces, DP and DP* properties are equivalent. Thus by [7], $T$ is completely continuous. On the other hand, $\ell_{1}$ is not a Grothendieck space and Grothendieck property is carried by complemented subspaces. Hence the Grothendieck space $X$ does not have any complemented copy of $\ell_{1}$. Since $X$ is a Banach lattice, by [12], it has the RDP property and so the completely continuous operator $T: X \rightarrow Y$ is weakly compact. Thus $X$ has the $L$-limited property, thanks to Theorem 2.8 .

As a corollary, since $\ell_{\infty}$ is a Banach lattice that has Grothendieck and DP properties, it has the $L$-limited property. This shows that the L-limited property on Banach spaces is not hereditary, since $c_{0}$ does not have this property. In the following, we show that the L-limited property is carried by every complemented subspace.

Theorem 2.12. If a Banach space $X$ has the L-limited property, then every complemented subspace of $X$ has the L-limited property.

Proof. Consider a complemented subspace $X_{0}$ of $X$ and a projection map $P: X \rightarrow X_{0}$. Suppose $T: X_{0} \rightarrow \ell_{\infty}$ is a limited completely continuous operator, so TP:X $\rightarrow \ell_{\infty}$ is also lcc. Since $X$ has the $L$-limited property, by Theorem 2.8, TP is weakly compact. Hence $T$ is weakly compact.

As another corollary, for infinite compact Hausdorff space $K$, we have the following corollary for the Banach space $C(K)$ of all continuous functions on $K$ with supremum norm.

Corollary 2.13. $C(K)$ has the L-limited property if and only if it contains no complemented copy of $c_{0}$.

Proof. We know that $C(K)$ is a Banach lattice with the DP property. On the other hand, $C(K)$ is a Grothendieck space if and only if it contains no complemented copy of $c_{0}$ [13]. So the direct implication is an application of Theorem 2.12 and the opposite implication is also an easy conclusion of Theorem 2.11. 


\section{Complementation in Lcc Operators}

In [11], Bahreini investigated the complementability of $W\left(X, \ell_{\infty}\right)$ and $C c\left(X, \ell_{\infty}\right)$ in $L\left(X, \ell_{\infty}\right)$. She showed that if $X$ is not a reflexive Banach space, then $W\left(X, \ell_{\infty}\right)$ is not complemented in $L\left(X, \ell_{\infty}\right)$ and if $X$ is not a Schur space, $C_{c}\left(X, \ell_{\infty}\right)$ is not complemented in $L\left(X, \ell_{\infty}\right)$. In the following, we investigate the complementability of $W\left(X, \ell_{\infty}\right)$ and $C c\left(X, \ell_{\infty}\right)$ in $\operatorname{Lcc}\left(X, \ell_{\infty}\right)$. We need the following lemma of [14].

Lemma 3.1. Let $X$ be a separable Banach space, and $\phi: \ell_{\infty} \rightarrow L\left(X, \ell_{\infty}\right)$ is a bounded linear operator with $\phi\left(e_{n}\right)=0$ for all $n$. Then there is an infinite subset $M$ of $\mathbb{N}$ such that for each $\alpha \in \ell_{\infty}(M)$, $\phi(\alpha)=0$, where $\ell_{\infty}(M)$ is the set of all $\alpha=\left(\alpha_{n}\right) \in \ell_{\infty}$ with $\alpha_{n}=0$ for each $n \notin M$.

Theorem 3.2. If $X$ does not have the L-limited property, then $W\left(X, \ell_{\infty}\right)$ is not complemented in $\operatorname{Lcc}\left(X, \ell_{\infty}\right)$.

Proof. Consider a subset $A$ of $X^{*}$ that is $L$-limited but it is not relatively weakly compact. So there is a sequence $\left(x_{n}^{*}\right)$ in $A$ that has no weakly convergent subsequence. Hence $S: X \rightarrow \ell_{\infty}$ defined by $S(x)=\left(\left\langle x, x_{n}^{*}\right\rangle\right)$ is an lcc operator but it is not weakly compact. Choose a bounded sequence $\left(x_{n}\right)$ in $B_{X}$ such that $S\left(x_{n}\right)$ has no weakly convergent subsequence. Let $X_{0}=\left[x_{n}\right]$, the closed linear span of the sequence $\left(x_{n}\right)$ in $X$. It follows that $X_{0}$ is a separable subspace of $X$ such that $S_{\left.\right|_{X_{0}}}$ is not a weakly compact operator. If $y_{n}^{*}=x_{n \mid X_{0}}^{*}$, we have $\left(y_{n}^{*}\right) \subseteq X_{0}^{*}$ is bounded and has no weakly convergent subsequence.

Now define $T: \ell_{\infty} \rightarrow \operatorname{Lcc}\left(X, \ell_{\infty}\right)$ by $T(\alpha)(x)=\left(\alpha_{n}\left\langle x, x_{n}^{*}\right\rangle\right)$, where $x \in X$ and $\alpha=$ $\left(\alpha_{n}\right) \in \ell_{\infty}$. Then

$$
\|T(\alpha)(x)\|=\sup _{n}\left|\alpha_{n}\left\langle x, x_{n}^{*}\right\rangle\right| \leq\|\alpha\| \cdot\left\|x_{n}^{*}\right\| \cdot\|x\|<\infty .
$$

We claim that for each $\alpha=\left(\alpha_{n}\right) \in \ell_{\infty}, T(\alpha) \in \operatorname{Lcc}\left(X, \ell_{\infty}\right)$.

Fix $\alpha=\left(\alpha_{n}\right) \in \ell_{\infty}$ and a weakly null and limited sequence $\left(x_{m}\right)$ in $X$. Since $\left(x_{n}^{*}\right)$ is an $L$-limited set, $\sup _{n}\left|\left\langle x_{m}, x_{n}^{*}\right\rangle\right| \rightarrow 0$. So we have

$$
\left\|T(\alpha)\left(x_{m}\right)\right\|=\sup _{n}\left|\alpha_{n}\left\langle x_{m}, x_{n}^{*}\right\rangle\right| \leq\|\alpha\|_{n}\left|\left\langle x_{m}, x_{n}^{*}\right\rangle\right| \longrightarrow 0,
$$

as $m \rightarrow \infty$. This finishes the proof of the claim and so $T$ is a well-defined operator into $\operatorname{Lcc}\left(X, \ell_{\infty}\right)$.

Let $R: \operatorname{Lcc}\left(X, \ell_{\infty}\right) \longrightarrow \operatorname{Lcc}\left(X_{0}, \ell_{\infty}\right)$ be the restriction map and define

$$
\phi: \ell_{\infty} \longrightarrow \operatorname{Lcc}\left(X_{0}, \ell_{\infty}\right) \text { by } \phi=R T
$$

Now suppose that $W\left(X, \ell_{\infty}\right)$ is complemented in $\operatorname{Lcc}\left(X, \ell_{\infty}\right)$ and

$$
P: \operatorname{Lcc}\left(X, \ell_{\infty}\right) \longrightarrow W\left(X, \ell_{\infty}\right)
$$


is a projection. Define $\psi: \ell_{\infty} \rightarrow W\left(X_{0}, \ell_{\infty}\right)$ by $\psi=R P T$. Note that as $T\left(e_{n}\right)$ is a rank one operator, we have $T\left(e_{n}\right) \in W\left(X, \ell_{\infty}\right)$. Hence

$$
\psi\left(e_{n}\right)=R P T\left(e_{n}\right)=R T\left(e_{n}\right)=\phi\left(e_{n}\right)
$$

for all $n \in \mathbb{N}$. By Lemma 3.1, there is an infinite set $M \subseteq \mathbb{N}$ so that $\psi(\alpha)=\phi(\alpha)$ for all $\alpha \in$ $\ell_{\infty}(M)$. Thus $\phi\left(X_{M}\right)$ is a weakly compact operator. On the other hand, if $\left(e_{n}^{*}\right)$ is the standard unit vectors of $\ell_{1}$, for each $x \in X_{0}$ and each $n \in \mathbb{N}$, we have

$$
\left\langle\left(\phi\left(\chi_{M}\right)\right)^{*}\left(e_{n}^{*}\right), x\right\rangle=\left\langle x_{n}^{*}, x\right\rangle .
$$

Therefore $\left(\phi\left(\chi_{M}\right)\right)^{*}\left(e_{n}^{*}\right)=x_{n \mid X_{0}}^{*}=y_{n}^{*}$ for all $n \in M$. Thus $\left(\phi\left(X_{M}\right)\right)^{*}$ is not a weakly compact operator and neither is $\phi\left(\chi_{M}\right)$. This contradiction ends the proof.

Corollary 3.3. Let $X$ be a Banach space. Then the following are equivalent:

(a) $X$ has the L-limited property,

(b) $W\left(X, \ell_{\infty}\right)=\operatorname{Lcc}\left(X, \ell_{\infty}\right)$,

(c) $W\left(X, \ell_{\infty}\right)$ is complemented in $\operatorname{Lcc}\left(\mathrm{X}, \ell_{\infty}\right)$.

We conclude this paper with another complementation theorem. Recall from [11] that a closed operator ideal $\mathcal{O}$ has the property $(*)$ whenever $X$ is a Banach space and $S$ is not in $\mathcal{O}\left(X, \ell_{\infty}\right)$, then there is an infinite subset $M_{0} \subseteq \mathbb{N}$ such that $S_{M}$ is not in $\mathcal{O}\left(X, \ell_{\infty}\right)$ for all infinite subsets $M \subseteq M_{0}$, where $S_{M}: X \rightarrow \ell_{\infty}$ is the operator defined by $S_{M}(x)=\Sigma_{m \in M} e_{m}^{*}(S x) e_{m}$, for all $x \in X$.

Theorem 3.4. If a Banach space $X$ does not have the DP* property, then $C_{c}\left(X, \ell_{\infty}\right)$ is not complemented in $\operatorname{Lcc}\left(X, \ell_{\infty}\right)$.

Proof. By hypothesis, there is a weakly null sequence $\left(x_{m}\right)$ in $X$ that is not limited. So there exists a weak* null sequence $\left(x_{n}^{*}\right)$ in $X^{*}$ such that

$$
\lim _{m \rightarrow \infty} \sup _{n}\left|\left\langle x_{m}, x_{n}^{*}\right\rangle\right| \neq 0 .
$$

Now define the operator $S: X \rightarrow \ell_{\infty}$ by $S(x)=\left(\left\langle x, x_{n}^{*}\right\rangle\right)$. By Theorem 2.2, $\left(x_{n}^{*}\right)$ is an $L$-limited set, but $S$ is not completely continuous. So for $X_{0}=\left[x_{n}\right],\left.S\right|_{X_{0}}$ is not completely continuous. Since $C_{c}\left(X_{0}, \ell_{\infty}\right)$ has the property $\left(^{*}\right)$ [11, Theorem 4.12], one can choose $M_{0} \subseteq \mathbb{N}$ so that for each infinite subset $M$ of $M_{0}, S_{M} \notin C c\left(X_{0}, \ell_{\infty}\right)$. Define $T: \ell_{\infty} \rightarrow \operatorname{Lcc}\left(X, \ell_{\infty}\right)$ by $T(\alpha)(x)=$ $\left(\alpha_{n}\left\langle x, x_{n}^{*}\right\rangle\right)$, where $x \in X$ and $\alpha=\left(\alpha_{n}\right) \in \ell_{\infty}$. As shown in the proof of the preceding theorem, $T$ is well defined.

Let $R: \operatorname{Lcc}\left(X, \ell_{\infty}\right) \rightarrow \operatorname{Lcc}\left(X_{0}, \ell_{\infty}\right)$ be the restriction map and define

$$
\phi: \ell_{\infty} \longrightarrow \operatorname{Lcc}\left(X_{0}, \ell_{\infty}\right) \text { by } \phi=R T
$$

Now suppose $C_{c}\left(X, \ell_{\infty}\right)$ is complemented in $\operatorname{Lcc}\left(X, \ell_{\infty}\right)$ and

$$
P: \operatorname{Lcc}\left(X, \ell_{\infty}\right) \longrightarrow \operatorname{Cc}\left(X, \ell_{\infty}\right)
$$


is a projection. Define $\psi: \ell_{\infty} \rightarrow C c\left(X_{0}, \ell_{\infty}\right)$ by $\psi=R P T$. Since

$$
\psi\left(e_{n}\right)=R P T\left(e_{n}\right)=R T\left(e_{n}\right)=\phi\left(e_{n}\right),
$$

for all $n \in \mathbb{N}$, one can use Lemma 3.1 to select an infinite subset $M$ of $M_{0}$ such that $\psi(\alpha)=$ $\phi(\alpha)$ for all $\alpha \in \ell_{\infty}(M)$. Thus $\phi(\alpha)=R T(\alpha)$ belongs to $C_{c}\left(X_{0}, \ell_{\infty}\right)$ for each $\alpha \in \ell_{\infty}(M)$. But $\left.T\left(X_{M}\right)\right|_{X_{0}}=S_{M} \notin C c\left(X_{0}, \ell_{\infty}\right)$, so we have a contradiction.

Corollary 3.5. Let X be a Banach space. Then the following are equivalent:

(a) X has the DP* property,

(b) $\operatorname{Cc}\left(X, \ell_{\infty}\right)=\operatorname{Lcc}\left(X, \ell_{\infty}\right)$,

(c) $C_{c}\left(X, \ell_{\infty}\right)$ is complemented in $\operatorname{Lcc}\left(X, \ell_{\infty}\right)$.

\section{References}

[1] J. Borwein, M. Fabian, and J. Vanderwerff, "Characterizations of Banach spaces via convex and other locally Lipschitz functions," Acta Mathematica Vietnamica, vol. 22, no. 1, pp. 53-69, 1997.

[2] E. Bator, P. Lewis, and J. Ochoa, "Evaluation maps, restriction maps, and compactness," Colloquium Mathematicum, vol. 78, no. 1, pp. 1-17, 1998.

[3] G. Emmanuele, "On Banach spaces with the Gelfand-Phillips property, III," Journal de Mathématiques Pures et Appliquées, vol. 72, no. 3, pp. 327-333, 1993.

[4] G. Emmanuele, "On the reciprocal Dunford-Pettis property in projective tensor products," Mathematical Proceedings of the Cambridge Philosophical Society, vol. 109, no. 1, pp. 161-166, 1991.

[5] I. Ghenciu and P. Lewis, "Dunford-Pettis properties and spaces of operators," Canadian Mathematical Bulletin, vol. 52, no. 2, pp. 213-223, 2009.

[6] I. Ghenciu and P. Lewis, "The Dunford-Pettis property, the Gelfand-Phillips property, and L-sets," Colloquium Mathematicum, vol. 106, no. 2, pp. 311-324, 2006.

[7] M. Salimi and S. M. Moshtaghioun, "The Gelfand-Phillips property in closed subspaces of some operator spaces," Banach Journal of Mathematical Analysis, vol. 5, no. 2, pp. 84-92, 2011.

[8] J. Diestel, Sequences and Series in Banach Spaces, vol. 92 of Graduate Texts in Mathematics, Springer, Berlin, Germany, 1984.

[9] R. E. Megginson, An Introduction to Banach Space Theory, vol. 183 of Graduate Texts in Mathematics, Springer, New York, NY, USA, 1998.

[10] L. Drewnowski, "On Banach spaces with the Gelfand-Phillips property," Mathematische Zeitschrift, vol. 193, no. 3, pp. 405-411, 1986.

[11] M. Bahreini Esfahani, Complemented subspaces of bounded linear operators, Ph.D. thesis, University of North Texas, 2003.

[12] C. P. Niculescu, "Weak compactness in Banach lattices," Journal of Operator Theory, vol. 6, no. 2, pp. 217-231, 1981.

[13] P. Cembranos, " $C(K, E)$ contains a complemented copy of $c_{0}$," Proceedings of the American Mathematical Society, vol. 91, no. 4, pp. 556-558, 1984.

[14] N. J. Kalton, “Spaces of compact operators," Mathematische Annalen, vol. 208, pp. 267-278, 1974. 


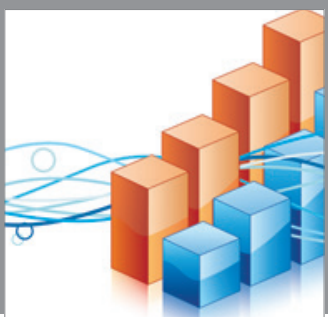

Advances in

Operations Research

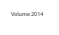

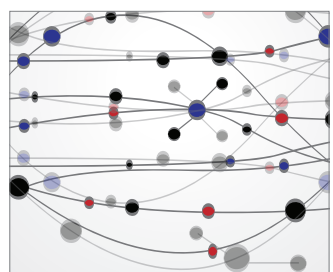

\section{The Scientific} World Journal
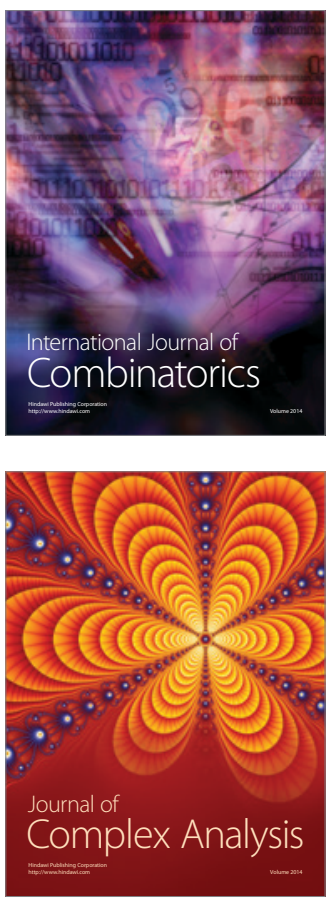

International Journal of

Mathematics and

Mathematical

Sciences
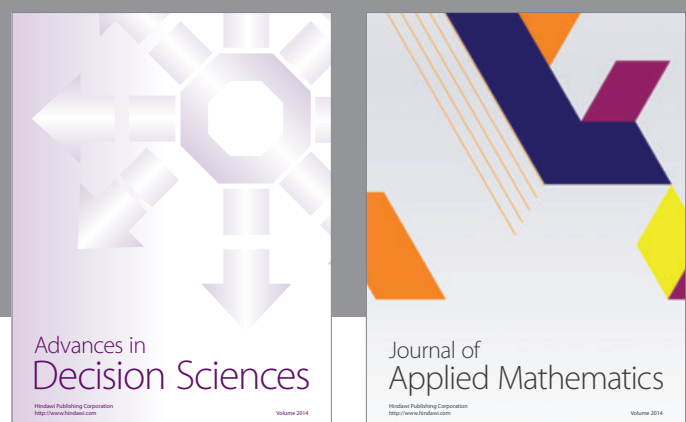

Journal of

Applied Mathematics
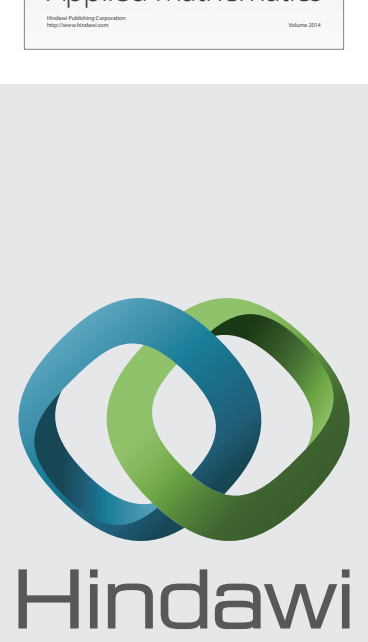

Submit your manuscripts at http://www.hindawi.com
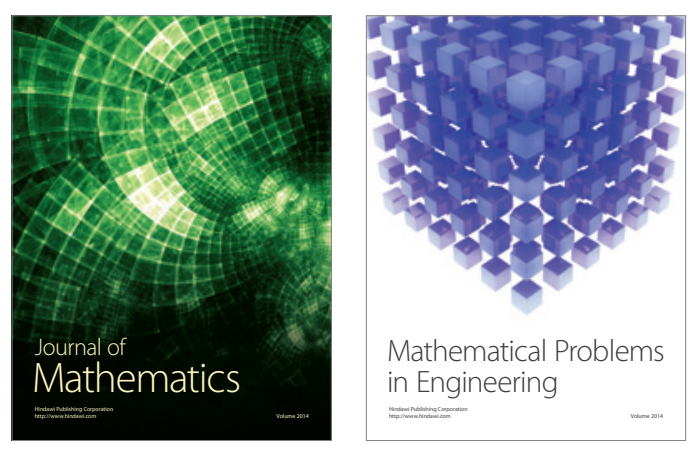

Mathematical Problems in Engineering
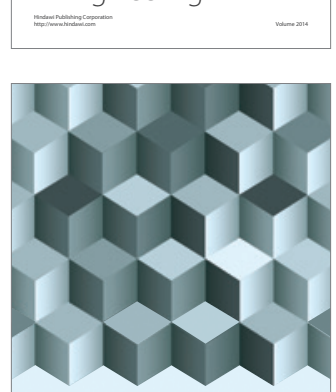

Journal of

Function Spaces
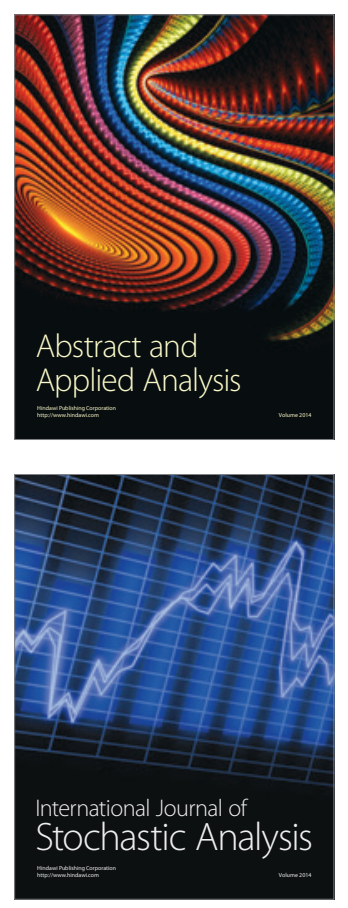

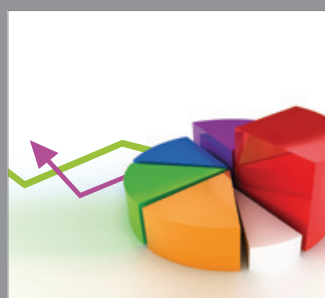

ournal of

Probability and Statistics

Promensencen
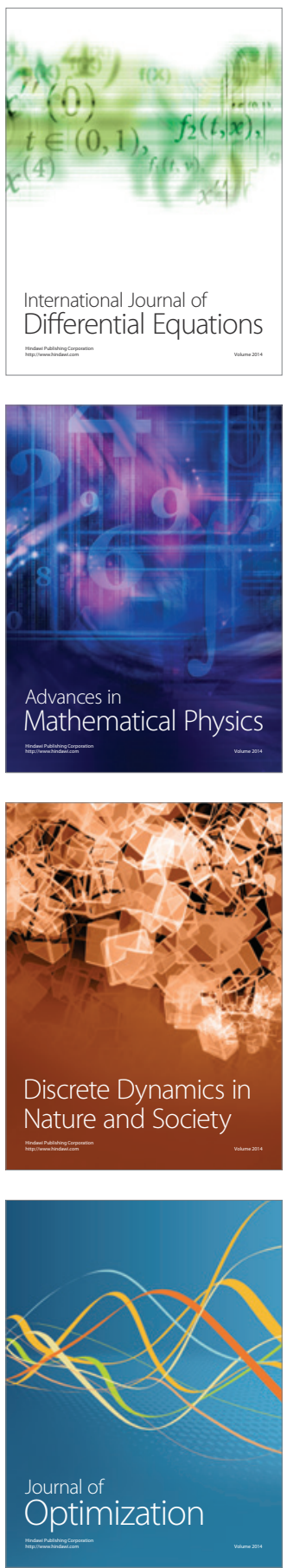